\section{Efeitos da maturação sexual na composição corporal, nos dermatóglifos, no somatótipo e nas qualidades físicas básicas de adolescentes}

\author{
Effects of sexual maturation on body composition, dermatoglyphics, \\ somatotype and basic physical qualities of adolescents
}

Renato Vidal Linhares ${ }^{1,2,4}$, Marcelo de Oliveira Matta², Jorge R. P. Lima², Paulo M. Silva Dantas ${ }^{2}$, Mônica Barros Costa ${ }^{3}$, José Fernandes Filho ${ }^{4}$

\section{RESUMO}

Objetivos: Descrever como se comportam a composição corporal, o somatótipo, as qualidades físicas básicas, os dermatóglifos e a idade óssea, nos diversos estágios de maturação sexual. Métodos: Foi realizado estudo transversal envolvendo 136 meninos, na faixa etária de 10 a 14 anos, e realizados exame clínico, avaliação física e radiografias de mãos e punhos para avaliação de idade óssea. Resultados: Encontraram-se tendência de aumento de estatura, massa corporal total, índice de massa corporal, idade óssea, diâmetros ósseos, circunferências musculares e qualidades físicas básicas, com o avançar da puberdade. Não se observaram diferenças nos dermatóglifos e no somatótipo, entre os estágios puberais. Conclusões: Por causa das mudanças em importantes parâmetros para o treinamento físico, durante a puberdade, a seleção de crianças e adolescentes para a atividade desportiva não deve se fundamentar apenas na idade cronológica, mas, sobretudo, na maturação sexual, visando a melhor avaliação física e o treinamento apropriado para essa população. Arq Bras Endocrinol Metab. 2009;53(1):47-54.

Descritores

Medicina do adolescente; puberdade; dermatoglifia; composição corporal; aptidão física

\begin{abstract}
Objectives: Describe the characteristics of body composition, somatotype, basic physical qualities, dermatoglyphics and bone age regarding sexual maturation stages of boys. Methods: A transversal study was carried out in 136 boys, between 10 and 14 years of age. Clinical assessment, physical examination and radiography of wrists and hands to calculate bone age were performed. Results: A tendency of increasing total body mass, stature, body mass index, body bone diameters and muscle circumferences and basic physical qualities was found with the advancing of puberty. No differences were found in dermatoglypics and somatotype between different stages of puberty maturation. Conclusions: Due to the changes in important parameters of physical training that occur during puberty, it can be concluded that the selection of children and adolescents for sportive training and competitions should be based not only on chronological age but also, and mainly on sexual maturation, for better physical assessment and appropriate training for this population. Arq Bras Endocrinol Metab. 2009;53(1):47-54.
\end{abstract}

Keywords

Adolescent medicine; puberty; dermatoglyphics; body composition; physical fitness

\author{
' Laboratório de Biociências \\ da Motricidade Humana, \\ Universidade Castelo Branco, Rio \\ de Janeiro, RJ, Brasil \\ ${ }^{2}$ Laboratório de Avaliação \\ Motora, Faculdade de Educação \\ Física e Desportos, Universidade \\ Federal de Juiz de Fora, MG, Brasil \\ ${ }^{3}$ Serviço de Endocrinologia, \\ Faculdade de Medicina, \\ Universidade Federal de \\ Juiz de Fora, MG, Brasil \\ ${ }^{4}$ Escola de Educação Física e \\ Desportos, Universidade Federal \\ do Rio de Janeiro, RJ, Brasil
}

\section{Correspondência para: Renato Vidal Linhares Rua João Afonso 11/407, Humaitá 22261-040 Rio de Janeiro, RJ renatolinharesjf@hotmail.com}

Recebido em 9/Ago/2007 Aceito em 10/Dez/2008

\section{INTRODUÇĀO}

$\mathrm{N}^{2}$ a adolescência, verifica-se que a maturação sexual exerce efeitos sobre o crescimento e o desenvolvimento, visto que grande variação maturacional pode ser encontrada entre adolescentes de mesma idade cronológica (1-2). Essa divergência entre maturação e idade cronológica é mais pronunciada quando se comparam meninos em estágios puberais contrastantes, ou seja, os mais atrasados e os mais avançados (3-7). 
Sem dúvida, a idade cronológica deve ser observada como indicador importante na caracterização de mudanças corporais, mas não a causa e o elemento principal. Isso leva a crer que possam estar sendo cometidos erros, tanto no grau de estimulação quanto nos métodos utilizados para o desenvolvimento de adolescentes de mesma idade cronológica, em atividades desportivas de escolas, clubes e centros esportivos, não apenas naquelas de cunho recreativo, mas, principalmente, nos processos de seleção, detecção e promoção de jovens atletas. Cargas externas mal dosadas podem levar a distúrbios, sobretudo, em crianças, nas quais estes estímulos podem causar lesões de maneira aguda ou subaguda, muitas vezes irreversíveis. Conhecer o momento em que se encontram crianças e adolescentes, dentro do processo de desenvolvimento, é importante instrumento para que o treinamento seja adequado, de acordo com o nível do aluno, e para que o professor possa adaptar as cargas às peculiaridades de cada um ( 1 ).

Há várias formas de se avaliar o desenvolvimento de crianças e adolescentes, merecendo destaque a avaliação esquelética, a análise do desenvolvimento dos caracteres sexuais, o estudo da idade dentária, além da avaliação somática (4).

A evolução da puberdade está associada a mudanças na composição corporal, na massa muscular e em vários componentes da aptidão física (8). Com base na observação de vários estudos é possível verificar que, no futebol, meninos com estágio puberal mais avançado são mais bem-sucedidos do que meninos menos desenvolvidos (9). No estudo de Macêdo e Fernandes Filho (10), em que o desenvolvimento puberal foi avaliado por meio das características sexuais secundárias, encontrou-se relação entre o desempenho atlético de adolescentes e a maturação sexual. A American Academy of Pediatrics e American Orthopaedic Society for Sports Medicine chegam, inclusive, a recomendar a avaliação do estadiamento puberal de crianças e adolescentes antes do início de treinamento de força, como medida de segurança diante de possíveis lesões musculoesqueléticas (11).

Com a caracterização fenotípica de determinado indivíduo, aos diversos aspectos citados anteriormente, ou seja, físicos, morfológicos e maturacionais, se junta o componente genético. Para a avaliação do potencial genético tem se utilizado a dermatoglifia, que permite não só a análise qualitativa, representada pelos padrões configuracionais, como também a análise quantitativa, representada pela contagem de linhas e índices formados por detalhes dermopapilares específicos (12). O estudo do potencial genético permite melhor aperfeiçoamento da preparação atlética e orientação esportiva antecipada e correta dos jovens, tendo em vista que diversos estudos apontam a existência de relação entre os padrões de dermatoglifia e as qualidades físicas (13-15).

O objetivo do presente estudo é avaliar a composição corporal, o somatótipo, as qualidades físicas básicas e os dermatóglifos, nos diversos estágios de maturação sexual, em crianças e adolescentes do sexo masculino.

\section{MÉTODOS}

Foi realizado estudo transversal que avaliou a composição corporal, a dermatoglifia, o somatótipo e as qualidades físicas básicas de escolares, do sexo masculino, na faixa etária de 10 a 14 anos. A amostra foi composta de indivíduos inscritos, em programa desportivo da Associação Municipal de Apoio Comunitário (AMAC), da cidade de Juiz de Fora, Minas Gerais, Brasil. Esse programa faz parte de um projeto social da prefeitura da cidade, no qual é oferecida a atividade futebol, meramente recreativa, com uma hora de duração, bissemanal, sob supervisão de treinador. No presente estudo, foram selecionados adolescentes inscritos por ocasião de sua admissão no programa, no ano de 2006. Participaram do estudo aqueles escolares devidamente matriculados, cujos responsáveis assinaram o termo de consentimento informado e esclarecido e foram excluídos os indivíduos que não compareceram nas datas marcadas para as avaliações e aqueles que apresentaram algum impedimento relacionado à saúde ou dificuldade em completar os testes físicos. Todos os indivíduos avaliados se encontravam em bom estado geral e não tinham diagnóstico de qualquer doença associada. O estudo foi aprovado pelo Comitê de Ética em Pesquisa da Universidade Federal de Juiz de Fora, MG, onde está registrado sob o número 474.159.2004-Grupo III.

Os indivíduos foram avaliados, em um primeiro momento, quanto a composição corporal, somatótipia e dermatoglifia. Para avaliação da composição corporal foram obtidos a massa corporal total (MCT), a estatura e o índice de massa corporal (IMC), calculado por meio da razão entre a MCT e a estatura elevada ao quadrado. As medidas da MCT e da estatura foram realizadas em balança de consultório com estadiômetro (Welmy® modelo 110), utilizando-se aproximação de 100 gramas para a MCT e de meio centímetro para a estatura. Os indivíduos eram avaliados sem sapatos e portando roupas leves.

Para o cálculo do somatótipo foi utilizado o método antropométrico de Heath e Carter (16), que necessita de dez medidas: MCT, estatura total, quatro dobras cutâneas (tríceps, subescapular, supraespinhal e panturrilha medial), 
dois diâmetros ósseos (biepicondiliano do úmero e biepicondiliano do fềmur) e dois perímetros (braço flexionado e perna medial). Para medida das dobras cutâneas foi utilizado adipômetro científico (Sanny®) e para aferição dos diâmetros ósseos utilizou-se paquímetro pequeno (Sanny®). Para aferir as circunferências do braço e da perna foi usada trena antropométrica (Sanny®).

Para a coleta dos dermatóglifos utilizou-se almofada coletora de impressões digitais (Impress ${ }^{\circledR}$ ) e papel branco rugoso. Foi obtida a impressão dos dez dedos das mãos e, posteriormente, realizadas as suas análises. Nas impressões digitais foram identificados os três tipos de desenho: (a) $\operatorname{arco}$ ou $\operatorname{arch}(\mathrm{A})$, que representa a ausência de deltas, sendo composto de cristas que atravessam transversalmente a almofada digital; (b) presilha ou loop (L), que corresponde ao feixe de linhas paralelas que faz uma volta de 180 graus e que possui sempre um delta e (c) verticilo ou whorl $(\mathrm{W})$, sistema nuclear de linhas formando círculos concêntricos ou espirais, que possui dois deltas, um de cada lado (17). De mais a mais, foram calculados a quantidade total de deltas (Dl0), que se refere à soma dos deltas dos dez dedos, e o somatório da quantidade total de linhas (SQTL), que corresponde ao total de linhas, nos dez dedos das mãos. A contagem das linhas foi realizada traçando-se uma linha que une o delta ao centro do sistema nuclear, visto que nessa contagem não se incluem as linhas que constituem o centro do sistema nuclear e do delta. No desenho tipo arco, não se faz a contagem de linhas, pois ele não possui delta, e nos desenhos verticilos são feitas duas contagens, uma para cada delta, sendo o número de linhas a média das duas contagens. Se o resultado final for ímpar, soma-se 0,5 ponto à média.

Em um segundo momento, foram avaliadas as qualidades físicas básicas. Os testes físicos foram realizados em quadra poliesportiva (força, velocidade e agilidade) e ao redor de um campo de futebol (potência aeróbica). Foram utilizados o teste de impulsão horizontal proposto por Johnson e Nelson (18), o teste de corrida de 30 metros de Popov (19), o teste Shuttle Run, $10 \times 5$ metros (20) e o teste de corrida de 1.000 metros de Klissouras (21), para avaliação da força, da velocidade, da agilidade e da potência aeróbica, respectivamente. O teste de impulsão horizontal de Johnson e Nelson é realizado com o indivíduo partindo da posição ortostática, com os pés paralelos e em pequeno afastamento lateral. Partindo de trás de uma linha de partida, o indivíduo que está sendo testado deverá saltar o mais longe possível, podendo contar com a ajuda da flexão das pernas e balanço dos braços. O resultado corresponde a distância entre a linha de partida e o calcanhar que tenha aterrizado o mais próximo da linha de partida. São permi- tidas três tentativas e computa-se o melhor resultado. O teste de corrida de 30 metros de Popov consiste da realização de uma corrida partindo da posição ortostática, atrás de uma linha de partida. Após os comandos de "pronto" e "vai", o indivíduo testado deverá correr, na maior velocidade possível, até outra linha de chegada demarcada a 30 metros de distância. Um dos testadores deverá abaixar seu braço para que o outro testador que está posicionado na linha de chegada possa acionar o cronômetro. A contagem de tempo é interrompida quando a maior parte do corpo do testando tiver ultrapassado a linha de chegada, sendo o teste realizado em uma única tentativa. O teste Shuttle Run $10 \times 5$ metros se inicia com o testador dando o sinal de "prepara" e, posteriormente, "vai", ao testando. O testando deverá correr a distância de 5 metros, durante cinco ciclos de idas e vindas, ou seja, 10 vezes a distância de 5 metros. Os locais de partida e chegada são demarcados com uma linha e dois cones, para limitação do espaço e melhor observação do testando que deverá ultrapassar, com ambos os pés, em cada passagem, as linhas de saída e chegada. $\mathrm{O}$ indivíduo terá duas chances para a realização do teste $\mathrm{e}$ será computado o melhor resultado. $\mathrm{O}$ teste de corrida de 1.000 metros, de Klissouras é realizado por meio de uma corrida de 1.000 metros, de maneira contínua, não sendo permitido caminhar durante o teste, sendo realizada apenas uma tentativa. $\mathrm{O}$ tempo de corrida $(\mathrm{Y})$ é medido em segundos e inserido na fórmula: $652,17-\mathrm{Y} \div 6,762$, para se encontrar o resultado final. Para as marcações dos testes físicos foi utilizada trena antropométrica (Sanny®).

Finalmente, os indivíduos compareceram ao Hospital Universitário da Universidade Federal de Juiz de Fora (HU-UFJF) para avaliação clínica e exame radiológico. Foi realizado exame clínico geral com ênfase na caracterização do desenvolvimento puberal. De cada indivíduo foi realizada avaliação da distribuição dos pelos pubianos e do estágio de desenvolvimento da genitália externa, utilizando-se os critérios de Marshall e Tanner (22). Nesse mesmo dia, foi realizada radiografia de mãos e punhos para determinação da idade óssea, utilizando-se os critérios de Greulich e Pyle (23).

Para análise dos dados, foi realizado tratamento estatístico descritivo contendo média e desvio-padrão, para idade cronológica, MCT, estatura, IMC, variáveis dermatoglíficas, diâmetros ósseos, circunferências, variáveis somatotípicas e qualidades físicas básicas. Para verificar a diferença entre os estágios puberais quanto aos parâmetros avaliados, utilizou-se estatística inferencial do estudo, tendo como teste a análise de variância. Quando a diferença foi significativa, procedeu-se ao teste de Scheffé. O nível de significância assumido foi de $5 \%(\mathrm{p}<0,05)$. 


\section{RESULTADOS}

Foram avaliados 136 escolares, do sexo masculino, na faixa etária de 10 a 14 anos, que foram divididos em categorias de acordo com o estágio da puberdade, adotando-se como critério a avaliação do desenvolvimento da genitália externa. A média, o desvio-padrão e a mediana da idade cronológica, nos diferentes estágios foram: no estágio $1,11,0 \pm 1,0$ anos e 11 anos; no estágio $2,12,3 \pm 1,0$ e 12 anos; no estágio $3,12,0 \pm$ 1,1 e 13 anos; no estágio $4,13,6 \pm 0,8$ e 14 anos; e no estágio 5 foi $13,6 \pm 0,5$ e 14 anos, respectivamente.

Como esperado, observou-se aumento da estatura, da MCT e da idade óssea com o avanço da puberdade. As médias do IMC foram de $16,1 \pm 2,21 \mathrm{~kg} / \mathrm{m}^{2}$ nos indivíduos pré-puberes; $18,3 \pm 3,17 \mathrm{~kg} / \mathrm{m}^{2}$ no estágio $2 ; 18,1$ $\pm 2,5 \mathrm{~kg} / \mathrm{m}^{2}$ no estágio $3 ; 18,4 \pm 1,8 \mathrm{~kg} / \mathrm{m}^{2}$ no estágio 4 ; e $21,5 \pm 4,98 \mathrm{~kg} / \mathrm{m}^{2}$ no estágio 5 . Os resultados referentes à distribuição dos indivíduos em cada estágio de desenvolvimento puberal quanto a idade cronológica, estatura, MCT, IMC e idade óssea estão representados na Tabela 1.

Em relação aos diâmetros e às circunferências, assim como ocorreu com os parâmetros referentes ao crescimento (MCT e estatura), observou-se aumento destes com o avanço da puberdade (Tabela 1 ).

Quanto ao somatótipo, não houve diferença estatisticamente significante, com a evolução da puberdade. Na endomorfia, houve crescimento nos valores do estágio 1 para o 2 e do estágio 4 para o 5 . Na mesomorfia, há tendência clara de aumento com o avançar da puberdade, com exceção da queda do estágio 3 para o 4 (Tabela 2).

\begin{tabular}{|c|c|c|c|c|c|c|}
\hline \multirow{2}{*}{$\begin{array}{l}\text { Padrões do } \\
\text { somatótipo }\end{array}$} & \multicolumn{6}{|c|}{ Estágios de desenvolvimento puberal } \\
\hline & $\mathbf{I}$ & II & III & IV & v & p \\
\hline Endomoria & $2,4 \pm 1,4$ & $3,6 \pm 2,2$ & $2,9 \pm 1,9$ & $2,2 \pm 1,7$ & $3,2 \pm 2,2$ & 0,050 \\
\hline Mesomorfia & $3,8 \pm 0,8$ & $4,0 \pm 1,2$ & $4,1 \pm 1,2$ & $3,5 \pm 0,6$ & $4,3 \pm 1,8$ & 0,480 \\
\hline Ectomorfia & $4,0 \pm 1,3$ & $3,4 \pm 1,6$ & $3,6 \pm 1,4$ & $4,1 \pm 1,4$ & $3,2 \pm 1,6$ & 0,122 \\
\hline
\end{tabular}

Quanto às qualidades físicas básicas, o desenvolvimento puberal exerceu influência na maioria dos parâmetros, com melhora dos resultados na força, na potência aeróbica e na velocidade, com o avanço da puberdade, em todos os estágios. É possível observar, por meio dos gráficos A, B, $\mathrm{C}$ e $\mathrm{D}$, a tendência de melhora nos resultados físicos dos adolescentes, com o avançar maturacional. Deve ficar claro que, na velocidade e na agilidade, a linha é decrescente, uma vez que o menor tempo na realização dos testes significa melhora nos resultados (Figura 1).

$\mathrm{Na}$ dermatoglifia, embora se observe tendência ao aumento do número de arcos, com o avançar da puberdade, com exceção do estágio 5 , não se obteve diferença estatisticamente significante. O número total de linhas dos dez dedos das mãos (SQTL) mostrou variação semelhante aos desenhos digitais. Na Tabela 3, estão representadas as variações nos tipos de desenho das digitais $(\mathrm{A}, \mathrm{L}, \mathrm{W})$ nos diferentes estágios da puberdade.

Tabela 1. Idade cronológica, estatura, massa corporal total (MCT), índice de massa corporal (IMC), idade óssea, diâmetros ósseos e circunferências musculares em relação aos estágios puberais (média \pm desvio-padrão) em escolares inscritos em programa desportivo da cidade de Juiz de Fora, MG.

\begin{tabular}{|c|c|c|c|c|c|c|}
\hline \multirow{2}{*}{ Parâmetros clínicos } & \multicolumn{6}{|c|}{ Estágios de desenvolvimento puberal } \\
\hline & $\begin{array}{c}\text { I } \\
(55)\end{array}$ & $\begin{array}{c}\text { II } \\
(40)\end{array}$ & $\begin{array}{c}\text { III } \\
(23)\end{array}$ & $\begin{array}{l}\text { IV } \\
(6)\end{array}$ & $\begin{array}{c}\text { V } \\
(12)\end{array}$ & $\begin{array}{c}p \\
(<0,05)\end{array}$ \\
\hline Idade cronológica (anos) & $11,0 \pm 1,0$ & $12,3 \pm 1,0$ & $12,0 \pm 1,1$ & $13,6 \pm 0,8$ & $13,6 \pm 0,5$ & $<0,05$ \\
\hline Idade óssea (anos) & $9,4 \pm 1,6$ & $11,4 \pm 1,6$ & $12,3 \pm 1,4$ & $13,7 \pm 0,4$ & $14,5 \pm 1,5$ & $<0,05$ \\
\hline Estatura (cm) & $141,9 \pm 6,1$ & $149,9 \pm 6,8$ & $152,8 \pm 5,4$ & $163,5 \pm 9,1$ & $169,3 \pm 5,0$ & $<0,05$ \\
\hline Massa corporal total (kg) & $32,7 \pm 5,70$ & $41,3 \pm 8,4$ & $42,4 \pm 6,4$ & $49,1 \pm 4,7$ & $61,9 \pm 14,4$ & $<0,05$ \\
\hline IMC $\left(\mathrm{kg} / \mathrm{m}^{2}\right)$ & $16,1 \pm 2,2$ & $18,3 \pm 3,2$ & $18,1 \pm 2,5$ & $18,4 \pm 1,8$ & $21,5 \pm 5,0$ & $<0,05$ \\
\hline Diâmetro do cotovelo (cm) & $5,3 \pm 0,3$ & $5,7 \pm 0,4$ & $5,9 \pm 0,3$ & $6,2 \pm 0,3$ & $6,4 \pm 0,5$ & $<0,05$ \\
\hline Diâmetro do joelho (cm) & $8,3 \pm 0,5$ & $8,8 \pm 0,5$ & $8,9 \pm 0,5$ & $9,2 \pm 0,4$ & $9,7 \pm 0,8$ & $<0,05$ \\
\hline Circunferência do braço (cm) & $21,7 \pm 2,0$ & $23,8 \pm 2,7$ & $23,3 \pm 3,9$ & $25,8 \pm 1,0$ & $27,8 \pm 3,3$ & $<0,05$ \\
\hline Circunferência da perna (cm) & $28,6 \pm 2,3$ & $30,6 \pm 2,8$ & $31,6 \pm 2,7$ & $32,2 \pm 1,6$ & $35,7 \pm 3,5$ & $<0,05$ \\
\hline
\end{tabular}


Tabela 3. Variáveis de dermatoglifia em relação aos estágios puberais (média \pm desvio-padrão) em escolares inscritos em programa desportivo da cidade de Juiz de Fora, MG.

\begin{tabular}{|c|c|c|c|c|c|c|}
\hline \multirow{2}{*}{ Variáveis de dermatoglifia } & \multicolumn{6}{|c|}{ Estágios de desenvolvimento puberal } \\
\hline & $\mathbf{I}$ & II & III & IV & $\mathbf{v}$ & $\mathbf{p}$ \\
\hline Arcos & $0,5 \pm 1,3$ & $0,5 \pm 1,2$ & $0,6 \pm 1,5$ & $1,1 \pm 2,0$ & $0,0 \pm 0,0$ & 0,428 \\
\hline Presilhas & $6,2 \pm 2,8$ & $6,3 \pm 3,0$ & $6,9 \pm 2,6$ & $4,8 \pm 3,3$ & $7,0 \pm 2,9$ & 0,507 \\
\hline Verticilos & $3,2 \pm 3,1$ & $3,2 \pm 3,2$ & $2,3 \pm 2,7$ & $4,0 \pm 4,1$ & $3,0 \pm 2,9$ & 0,758 \\
\hline D10 & $12,6 \pm 3,7$ & $12,7 \pm 3,8$ & $11,7 \pm 3,5$ & $12,8 \pm 5,6$ & $13,0 \pm 2,9$ & 0,811 \\
\hline SQTL & $121,1 \pm 39,6$ & $121,2 \pm 41,5$ & $119,5 \pm 37,8$ & $128,3 \pm 47,3$ & $125,5 \pm 30,7$ & 0,985 \\
\hline
\end{tabular}

A

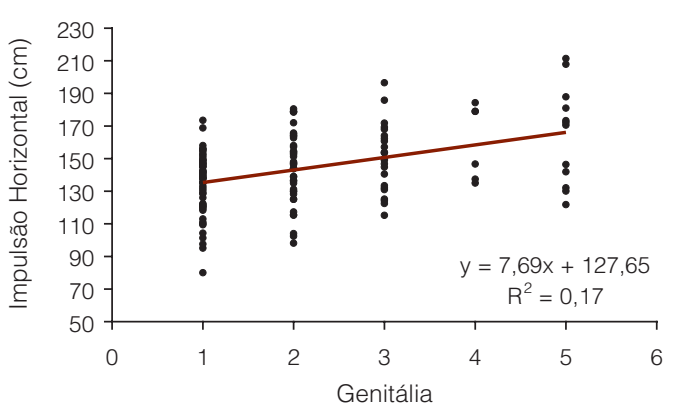

C

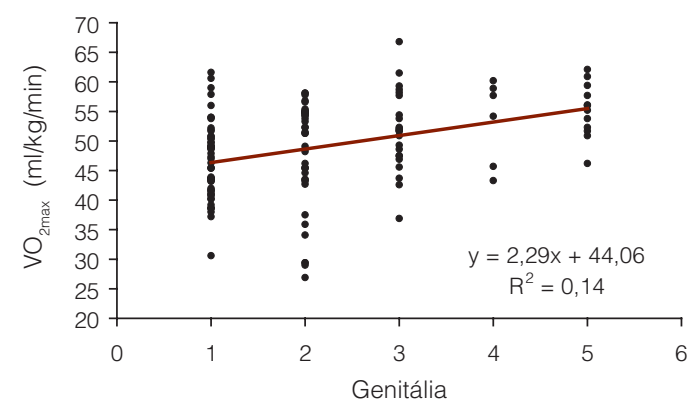

B

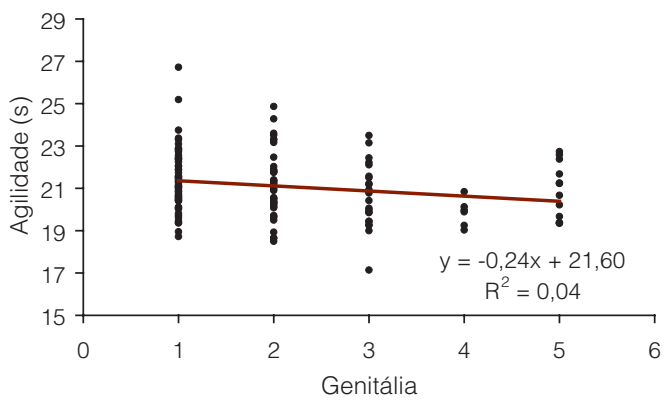

D

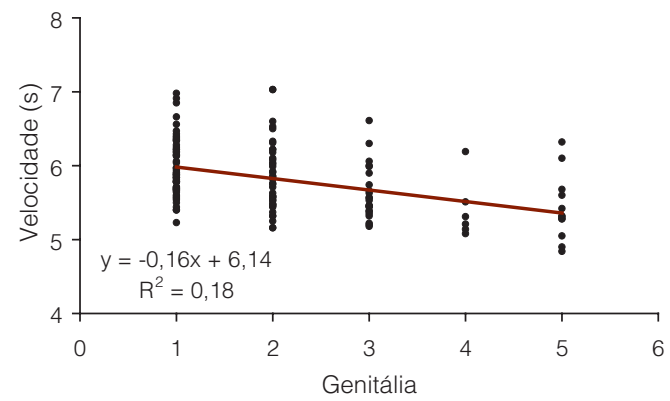

Figura 1. Relação entre força $(A)$, agilidade $(B), V_{2 \max }(C)$ e velocidade (D) e os estágios do desenvolvimento puberal em escolares inscritos em programa desportivo da cidade de Juiz de Fora, MG.

\section{DISCUSSÃO}

A estatura, a MCT e o IMC estão relacionados ao crescimento e desenvolvimento de crianças e adolescentes que possuem ritmos biológicos diferentes, principalmente na puberdade caracterizada por importantes transformações físicas que acompanham o desenvolvimento dos caracteres sexuais secundários (24). Os resultados do presente estudo apontam diferença significativa entre os estágios puberais na MCT, na estatura, no IMC e na idade óssea, fenômeno clássico na prática médica, mas que, muitas vezes, não é valorizado pelos profissionais ligados à prática desportiva. Nessa área, Malina (4) relata que jovens com maturação biológica precoce quando comparados a indivíduos com maturação tardia são, geralmente, mais pesados e mais altos para a idade. Da mesma forma, Freitas e cols. (25) relatam súbito aumento nos valores de MCT em um estudo com meninos de 7 a 18 anos. Klein e Fernandes Filho (26) também em um estudo com adolescentes, do sexo masculino, de 10 a 13 anos observaram tendência de aumento da idade, da MCT e da estatura entre indivíduos pré-púberes e púberes. Da mesma forma, Schneider, Rodrigues e Meyer (27) estudando meninos pré-púberes, púberes e póspúberes constataram tendência de aumento da idade, MCT, estatura e IMC com a evolução da puberdade. Tendo em vista que os dados do presente estudo corro- 
boram com aqueles da literatura, ao se utilizar a idade cronológica como parâmetro de seleção e caracterização de atletas, nas competições para crianças e adolescentes, agrupando-se as categorias em dois anos seguidos de nascimento, pode-se prejudicar ainda mais a avaliação do desempenho desse grupo de indivíduos.

No presente estudo, observou-se, ainda, que embora houvesse aumento na MCT e na estatura com a progressão da maturação sexual, os valores do IMC mostraram diminuição do estágio 2 para o estágio 3 , o que poderia estar relacionado ao aumento proporcionalmente maior da estatura sobre a MCT, nessa fase de crescimento muito acelerado, uma vez que, no estágio 4 os indivíduos voltaram ao padrão anterior de aumento dos parâmetros antropométricos. Sabe-se que o estágio 3 de desenvolvimento da genitália masculina coincide com o início do estirão puberal, o pico máximo de velocidade de crescimento, e serve como auxílio na avaliação do estado nutricional (28). Barbosa, Franceschini e Priore (29) ressaltam, inclusive, que o estadiamento puberal e a maturação sexual devem ser analisados para melhor compreensão do estado nutricional de adolescentes, por interferir em modificações antropométricas e de composição corporal.

$\mathrm{Na}$ análise dos diâmetros ósseos do úmero e do fêmur e das circunferências do braço contraído e da panturrilha medial verificam-se diferenças significativas entre os estágios puberais, com aumento dos resultados, com o avançar da puberdade. Todavia, entre os estágios 2 e 3, foi encontrada queda, embora não significativa, nos valores da circunferência do braço contraído. Tais fatos voltam a demonstrar que adolescentes de mesma idade cronológica podem apresentar estruturas diferentes, por estarem passando por momentos diferentes, ou seja, enquanto alguns já estão na puberdade ou mesmo no final dela, outros ainda possuem características pré-puberais. Não há fase de mudanças mais intensas e rápidas na composição corporal do que na puberdade, o que deve merecer atenção especial por parte dos profissionais que trabalham no treinamento infanto-juvenil $(6-7,10)$.

Há preocupação por parte de professores e pesquisadores com a intensidade do treinamento com jovens, pois ele pode levar a transtornos de ordem articular, óssea, muscular, cardíaca e até mesmo psicológica por não suportarem a carga imposta (30-33). No somatótipo, não houve diferenças significativas entre os estágios puberais. Todavia, na endomorfia, que está relacionada aos depósitos de gordura, houve variação entre os estágios, com tendência à queda entre os estágios 2 e 4, de maneira semelhante àquela observada no IMC, índice que também guarda relação com a gordura corporal. Observou-se, também, aumento na endomorfia do estágio 1 para o 2 e do estágio 4 para o 5 , encontrando-se, na análise no post hoc, diferença estatisticamente significativa nas dobras cutâneas, que são utilizadas na fórmula de cálculo da endomorfia. Na mesomorfia, que se relaciona com os diâmetros ósseos e as circunferências musculares, houve tendência de aumento entre os estágios, o que seria esperado, na medida em que se observa tendência de aumento dos diâmetros e das circunferências com a puberdade. Na ectomorfia, que está ligada à estatura e à MCT, há variação semelhante àquela verificada na endomorfia. É compreensível esta não-linearidade no somatótipo, por se tratar de componente ligado não somente à genética, mas que também recebe influência de fatores ambientais, como hábitos alimentares e exercício físico, entre outros. Outrossim, o adolescente está em constante transformação, com momentos de maiores incrementos na MCT e momentos com maiores incrementos na estatura.

Nas qualidades físicas básicas, verificou-se diferença significativa com a progressão da puberdade, para a maioria dos testes utilizados. No salto horizontal, no qual o componente força exerce grande influência, a tendência de aumento com o avançar da maturação é esperado, tendo em vista que os indivíduos estão passando pela puberdade, quando ocorre aumento da secreção de esteróides sexuais. Como se sabe, os androgênios são responsáveis por grande parte das alterações físicas observadas na puberdade, na qual a aceleração do crescimento linear se acompanha de ganho de massa muscular, aumento de tecido conectivo e, como consequência, balanço nitrogenado positivo (34). Macêdo e Fernandes Filho (10) encontraram relação entre maior nível de força, medida por meio do salto vertical e o avançar da maturação sexual.

$\mathrm{Na}$ agilidade, não se encontrou diferença significativa entre os estágios puberais, embora seja possível perceber tendência à progressão. Para esse teste há necessidade de força, pois a velocidade está envolvida e, ao mesmo tempo, é preciso boa coordenação, para que as mudanças de posição e direção do corpo sejam feitas de maneira satisfatória. Entre os estágios 4 e 5 observou-se tendência à queda na agilidade, possivelmente relacionada à dificuldade de trabalhar ao mesmo tempo com a estatura e a troca de direção exigida pelo teste. Além disso, é preciso considerar que, além da puberdade, possa existir influência genética nos resultados dos testes físicos, já que foram encontradas diferenças entre os indivíduos no que diz respeito aos aspectos relacionados à coordenação, como maior número de deltas (D10) e do total de linhas (SQTL).

Na potência aeróbica e na velocidade, a tendência dos resultados se assemelha ao salto horizontal. Algumas diferenças nos resultados podem estar ligadas à dificuldade 
dos adolescentes em lidar com o crescimento desordenado dos segmentos corporais, típico da puberdade e, por isso mesmo, com a coordenação.

Diversos autores sugerem que o número de arcos está relacionado à maior dificuldade quanto aos fatores coordenativos, enquanto os desenhos do tipo presilha se ligam à maior potencialidade em termos de velocidade e os verticilos estão relacionados a atividades cíclicas. Outrossim, os aumentos do número de deltas e do número total de linhas estão relacionados à maior potencialidade coordenativa (13-15). De maneira geral, no presente estudo, a avaliação do potencial genético a partir das variáveis dermatoglíficas não mostrou diferença significativa entre os estágios puberais. Em concordância com alguns estudos, notou-se aumento de desenhos do tipo arco, com o avanço da puberdade, à exceção do estágio 5 , em que não houve indivíduo com este tipo de desenho digital, além de tendência de melhora do D10 e SQTL, que estão ligados à melhora no potencial coordenativo, achados também foram descritos no estudo de Klein e Fernandes Filho entre alunos pré-púberes e púberes (26).

Tendo em vista que a puberdade é uma fase de grandes e constantes mudanças, o conhecimento do maior número de variáveis e suas tendências em função do crescimento e desenvolvimento facilita a maior compreensão do momento em que se encontra o jovem e propicia trabalho mais efetivo com esse público. Com a utilização dos resultados do presente estudo na prática desportiva, podem ser evitados excessos e exageros, o que permite treinamento mais apropriado ao momento em que se encontra o adolescente. Além disso, pode ser possível tanto a inserção mais tranquila e adequada nas competições quanto a seleção de talentos mais fidedigna e consistente com o nível de desenvolvimento de cada indivíduo, levando a menor erro de prognóstico pelo privilégio que crianças e adolescentes de maturação precoce possuem.

Agradecimentos: Os autores agradecem aos professores Danilo, Beto, Natanael, Cláudio e Edvaldo pela permissão da realização do estudo e pelo apoio na organização das atividades com os alunos, facilitando o desenvolvimento do trabalho. Agradecem, também, aos alunos Victor e Rafael da Faculdade de Medicina da UFJF, pelo apoio na avaliação médica e na realização das radiografias para idade óssea.

Declaração: Os autores declaram não haver conflito de interesse científico neste artigo.

\section{REFERÊNCIAS}

1. Papalia DE, Olds SW, Feldman RD. Desenvolvimento humano. 8. ed. Porto Alegre: Artmed; 2006.

2. Borges JLC, Brandão CMA. Low bone mass in children and adolescents. Arq Bras Endocrinol Metab. 2006;50:775-82.
3. Philippaerts RM, Vaeyens R, Janssens M, Van Tenterghem B, Matthys D, Craen R, et al. The relationship between peak height velocity and physical performance in youth soccer players. J Sports Sci. 2006;24:221-31.

4. Malina RM. Crescimento, maturação e desempenho. In: Garret Jr. WE, Kirkendall DT, editores. A ciência do exercício e dos esportes. São Paulo: Artmed; 2003. p. 454-75.

5. Rowland TW. Development exercise physiology. $2^{a}$ ed. Champaing: Human Kinetics; 2005.

6. Malina RM, Brown EW. Growth and maturation of football players: implications for selection in youth programs. Insight: The F.A. Coaches Association J. 1998;2:27-30.

7. Seabra A, Catela D. Maturação, crescimento físico e prática esportiva em crianças. Horizonte. 2000;14:15-7.

8. Vaeyens R, Philippaerts RM, Malina RM. The relative age effect in soccer: a match-related perspective. J Sports Sci. 2005;23:747-57.

9. Malina RM, Reyes MEP, Eisenmann JC, Horta L, Rodrigues J, Miller R. Stature, mass and maturity. J Sports Sci. 2000;18:685-97.

10. Macêdo MM, Fernandes Filho J. Estudo das características dermatoglíficas, somatotípicas e das qualidades físicas básicas nos diversos estágios de maturação sexual. Fit Perf J. 2003;2:315-20.

11. Benjamin HJ, Glow KM. Strength training for children and adolescentes. Phys Sportsmed. 2003;31:23-33.

12. Fernandes Filho J, Abramova TF. Diagnóstico do potencial genético dos jogadores de voleibol do Brasil através das impressões digitais 1996 [acesso em 1996 May]. Disponível em http://www.castelobranco. br/prppg/stricto/fernandes/digitaisvolei.

13. Nogueira TN, Cunha Júnior AT, Silva Dantas PM, Fernandes Filho J. Perfil somatotípico, dermatoglífico e das qualidades físicas da seleção brasileira de handebol feminino adulto por posição de jogo. Fit Perf J. 2005;4:236-41.

14. Almeida MN, Fernandes Filho J, Silva Dantas PM. Relação dos índices dermatoglíficos com avaliação isocinética e ergoespirometria. Fit Perf J. 2005;4:101-7.

15. Arutiohian AG. Impressões digitais como meio de seleção e prognóstico no esporte. In: Coletânea de Teses do Simpósio - Rimelnintsk. Marcas genéticas na medicina e antropogenética; 1988:140.

16. Carter JEL, Heath BH. Somatotyping: Development and Aplications. Melksham: Cambridge University Press; 1990.

17. Cummins $\mathrm{H}$, Midlo C. Fingerprints, Palms and Soles an Introduction to Dermatoglyphics. Philadelphia: Blakiston; 1961.

18. Johnson BL, Nelson JK. Practical Measurements for Evaluation in Physical Education. Minnesota: Burges Publishing Company; 1979.

19. Marins JCB, Giannichi RS. Avaliação e Prescrição de Atividade Física: Guia Prático. 2ª ed. Rio de Janeiro: Shape; 1998.

20. Eurofit: ala bateria eurofit a Catalunya. Generalitat de Catalunya Department de la Presidència Secretaria General de I'Esport. Barcelona: Gratifiques Cromotip; 1993.

21. Fernandes Filho J. A prática da avaliação física: testes, medidas avaliação física em escolares, atletas e academias de ginástica. Rio de Janeiro: Shape; 2003.

22. Marshall WA, Tanner JM. Variations in pattern of pubertal changes in boys. Arch Dis Child. 1970;45:13-23.

23. Greulich WW, Pyle SI. Radiographic Atlas of Skeletal Development of the Hand and wrist. $2^{\underline{a}}$ ed. Stanford: Stanford University Press; 1959.

24. Zeferino AMB, Barros Filho AA, Bettiol H, Barbieri MA. Acompanhamento do crescimento. J Pediatr. 2003;79:23-32.

25. Freitas DL, Maia JA, Marques AT, Beunen GP, Lefevre JA, Claessens $A L$, et al. Studies in somatic growth, biological maturation, physical 
fitness and activity in portuguese speaking countries an overview. Port J Sport Sci. 2002;2:92-111.

26. Klein CMO, Fernandes Filho J. Relação entre a dermatoglifia, as qualidades físicas e o nível maturacional de escolares adolescentes de ambos os sexos. Fit Perf J. 2003;2:321-9.

27. Schneider P, Rodrigues LA, Meyer F. Dinamometria computadorizada como metodologia de avaliação da força muscular de meninos e meninas em diferentes estágios de maturação. Rev Paul Educ Fis. 2002;16:35-42.

28. WHO. Physical status: the use and interpretation of anthropometry. Geneve, 1995. (Technical Report Series, 854).

29. Barbosa KBF, Franceschini SCC, Priore SE. Influência dos estágios de maturação sexual no estado nutricional, antropometria e composição corporal de adolescentes. Rev Bras Saude Mater Infant. 2006;6:375-82.
30. Barbanti V, Tricoli V. A formação do esportista. In: Gaya A, Marques A, Tani G, editores. Desporto para crianças e jovens: razões e finalidades. Porto Alegre: UFRGS; 2004. p. 199-216.

31. Fujii K, Demura S, Matsuzawa J. Velocity of heigth by wavelet interpolation method in Japanese high school athletes. J Physiol Anthropol Appl Human Sci. 2005;24:15-22.

32. Michaud P-A, Renaud A, Narring F. Sports activities related to injuries? A survey among 9-19 year olds in Switzerland. Inj Prev. 2001;7:41-5.

33. America Academy of Pediatrics. Intensive Training and Sports Specialization in Young Athletes. Pediatrics. 2000;106(1):154-7.

34. Griffin JE, Wilson JD. Disorders of testes and the male reproductive tract. In: Wilson JD et al, editores. Williams Textbook of Endocrinology. Philadelphia: W.B. Saunders Company; 1998. p. 819-75. 